\title{
Hydration dynamics of oriented DNA films investigated by time-domain terahertz spectroscopy
}

\author{
C. Kistner, A. André, ${ }^{\text {a) }}$ T. Fischer, A. Thoma, C. Janke, A. Bartels, T. Gisler, \\ G. Maret, and T. Dekorsy) \\ Department of Physics, University of Konstanz, 78465 Konstanz, Germany \\ and Center for Applied Photonics, University of Konstanz 78465 Konstanz, Germany
}

\begin{abstract}
The $B$ to $A$ conformational transition of highly oriented DNA films due to a hydration change is observed with time-domain terahertz spectroscopy. Wet-spun films of calf thymus and salmon DNA are investigated for different film thicknesses and for different polarizations of the terahertz radiation relative to the DNA orientation. A clear polarization dependence is observed. Asynchronous optical sampling allows recording of terahertz absorption and background spectra in a few $10 \mathrm{~s}$, permitting the tracking of the dehydration dynamics on a time scale of minutes. The observation of a phase transition is corroborated by Raman spectroscopy.
\end{abstract}

The conformation of DNA is directly linked to important biological processes such as DNA replication and DNA repair. While DNA in aqueous solution is predominantly in the $B$ form, it is also frequently seen to deviate from this conformation. Dehydration of $B$-type DNA to below 30\% water content by weight converts it into $A$-type DNA. This conformational transition and its origins in terms of free energy difference between $A$ - and $B$-type conformations have been intensively studied theoretically ${ }^{1}$ and experimentally, e.g., by x-ray diffraction. ${ }^{2-4}$

Low-frequency vibrational excitations are expected to play an important role in conformational dynamics of DNA, as well as in the hydration shell, and might influence the dynamics of drug binding. ${ }^{5}$ In particular, Raman and Brillouin scattering ${ }^{6,7}$ as well as thermal neutron scattering ${ }^{8}$ have been used to observe low-frequency excitation spectra. Far-infrared absorption spectroscopy in the terahertz frequency range provides another access to the low-frequency vibrational excitations of DNA. Besides Fourier-transform spectroscopy ${ }^{9-11}$ and absorption spectroscopy using tunable terahertz light sources, ${ }^{12,13}$ time-domain terahertz spectroscopy became available recently to study optical properties of biomolecules and DNA. ${ }^{14-17}$

In this letter, we use a recently developed terahertz spectrometer based on high-speed asynchronous optical sampling (ASOPS) to measure the terahertz transmission characteristics of highly oriented wet-spun DNA films. We describe the dependences of the terahertz absorption in the range of $100 \mathrm{GHz}-2.0 \mathrm{THz}$ on the degree of hydration. Here, we take advantage of the fast sampling possibilities that are inherent to the ASOPS technique. The results from the terahertz experiments are corroborated by x-ray diffraction and Raman scattering experiments.

Highly oriented films are produced from DNA sodium salt (Sigma-Aldrich Co.) using the wet-spinning method developed by Rupprecht. ${ }^{18}$ A highly concentrated DNA solution is pumped through a spinneret and precipitated in $77 \%$

\footnotetext{
${ }^{a)}$ Also at: Université Louis Pasteur, 4 rue Blaise Pascal, 67070 Strasbourg Cedex, France.

${ }^{b)}$ Electronic mail: thomas.dekorsy@uni-konstanz.de
}

ethanol solution containing $0.3 \mathrm{M} \mathrm{NaCl}$ at a temperature of $5{ }^{\circ} \mathrm{C}$ such that thin DNA fibers are generated and spooled onto a rotating cylinder. Multilayered DNA films are taken off the cylinder and are dried at $5{ }^{\circ} \mathrm{C}$ and are rehydrated at $75 \%$ relative humidity (r.h.) at room temperature for at least three days. The orientation of the helix axis inside these films is very high. ${ }^{19}$ The crystallinity of the films was confirmed by x-ray diffraction. For the dehydration experiments, the samples were hydrated prior to the experiments overnight to ensure that the DNA inside the film had almost entirely reached $\mathrm{B}$ configuration. We observed the structure of the DNA films by x-ray diffraction before and after hydration, which shows diffraction patterns characteristic of the A and $\mathrm{B}$ form, respectively. However, the long data acquisition times in $\mathrm{x}$-ray diffraction prevent the investigation of the hydration dynamics on a time scale of minutes by x-ray diffraction. The thickness of the samples under investigation was determined with an accuracy of about $10 \%$ in the dehydrated state. For the salmon DNA and for the calf thymus DNA films thicknesses of about 25 and $70 \mu \mathrm{m}$, respectively, were measured.

The ASOPS terahertz spectrometer is described in detail elsewhere. ${ }^{20}$ It is based on two $1 \mathrm{GHz}$ Ti: sapphire femtosecond lasers (GigaJet Twin, Gigaoptics GmbH, Germany) which are linked at a fixed repetition rate difference of $10 \mathrm{kHz}$ by an electronic stabilization circuit. This technique enables us to achieve a high signal-to-noise ratio of approximately $50 \mathrm{~dB}$ in a short data acquisition time of $10 \mathrm{~s}$ which is essential for this study. One femtosecond laser $(600 \mathrm{~mW}$, $800 \mathrm{~nm}$ ) drives a high-efficiency large area GaAs-based terahertz emitter which emits linearly polarized terahertz radiation. ${ }^{21}$ The generated terahertz pulses are guided by four off-axis parabolic mirrors and focused onto a ZnTe crystal for electro-optical sampling. The polarized probe pulses provided by the second laser $(700 \mathrm{~mW}, 790 \mathrm{~nm})$ are focused on the detection crystal via a hole in the fourth off-axis parabolic mirror. The probe beam polarization is analyzed with a half-wave plate and a polarizing beam splitter. A single photoreceiver is used for the detection. The recorded polarization changes are a direct measure of the terahertz electric field in amplitude and phase. ${ }^{22}$ Spectral data are obtained by 


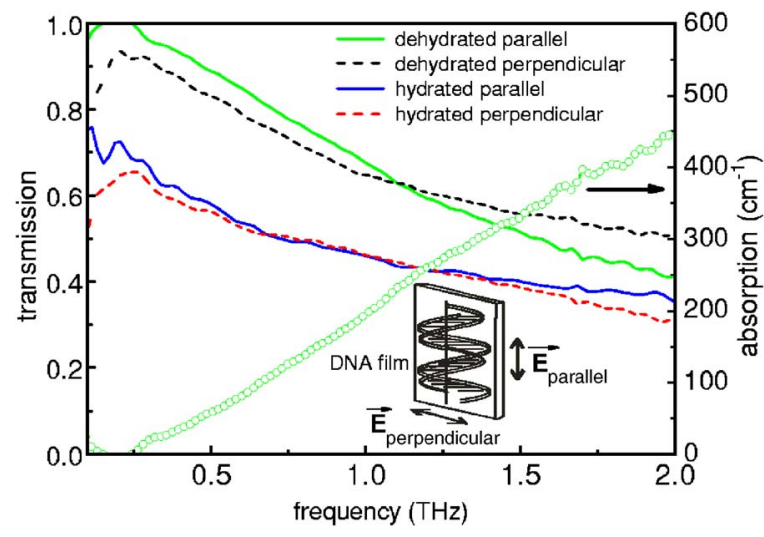

FIG. 1. (Color online) Transmission of hydrated and dehydrated salmon DNA films for different film orientations, as given in the figure. Right axis (dotted line): absorption for the dehydrated parallel case. The inset illustrates the orientation of the helix axis toward the polarization of the terahertz electric field.

Fourier transform of the electric field. For the Fourier transformation we use $14 \mathrm{ps}$ time delay of the total measured $1 \mathrm{~ns}$ time delay. This procedure reduces the spectral resolution of the spectra to approximately $70 \mathrm{GHz}$. Sharper spectral features are not present in the investigated samples which was checked by taking Fourier transforms over longer time delays.

Transmission spectra are obtained from a reference and a sample spectrum by moving the DNA film into and out of the terahertz focus for each point of the dehydration curve. To avoid absorption of the terahertz beam by ambient water vapor the whole setup was purged with dry air. The sample cell possesses holders to mount the film in different orientations towards the terahertz beam polarization. A reservoir in the sample cell contains saturated $\mathrm{K}_{2} \mathrm{SO}_{4}$ solution to adjust the relative humidity to about $98 \%$. The relative humidity is monitored with a humidity sensor. We utilize $100 \mu \mathrm{m}$ thick mylar foils as cell windows. Raman spectra were obtained with a spectrometer (Labram HR, Jobin Yvon) using a $532 \mathrm{~nm}$ green laser as excitation source. For the Raman measurements sample conditions similar to the ones in the terahertz measurements were established. Raman spectra in the range of $600-900 \mathrm{~cm}^{-1}$ were accumulated within $90 \mathrm{~s}$.

First, we investigated hydrated and dehydrated salmon DNA films for different orientations of the helix axis relative to the polarization of the terahertz radiation. Figure 1 shows the transmission spectra for parallel and perpendicular polarization of the terahertz field relative to the DNA orientation. The transmission through the dehydrated film is higher than that through the hydrated films for both polarization directions. The transmission decreases from $250 \mathrm{GHz}$ to $2.0 \mathrm{THz}$ for all films without showing sharp spectral features. The transmission spectra cover a range of absorption $\alpha$ from close to $0 \mathrm{~cm}^{-1}$ to $\alpha=450 \mathrm{~cm}^{-1}$ (shown for the dehydrated film and parallel polarization in Fig. 1). An uncertainty of about $10 \%$ arises for this quantity due to an uncertainty in the film thickness. Since the thickness dependence on the hydration state is not quantified, we plot transmission spectra instead of absorption spectra.

The difference of absorption between parallel and perpendicular polarization is larger in the dehydrated film than in the hydrated one. Only below $250 \mathrm{GHz}$ does the perpendicular polarization exhibit a significantly stronger absorp-
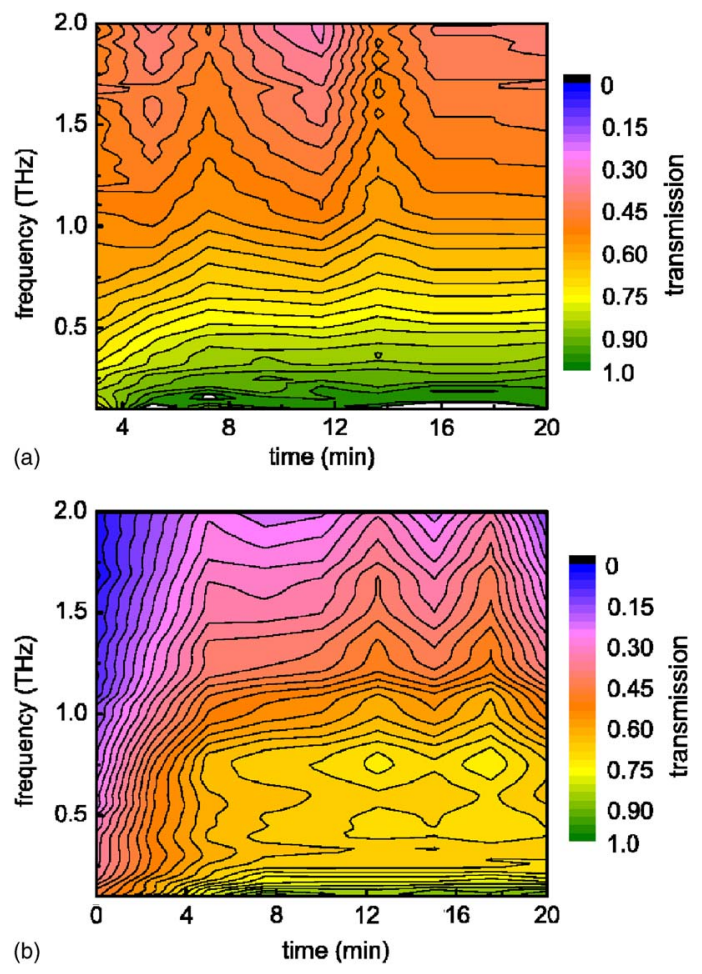

FIG. 2. (Color online) Contour plots of the temporal evolution of the transmission change for a salmon DNA film (a) and a calf thymus DNA film (b).

tion than the parallel polarization. The absence of stronger absorption for the parallel orientation shows that electronic conductivity along the DNA chains is negligible in this frequency range. ${ }^{23}$ Different hydrations of the DNA changes electrostatic interactions and van-der-Waals forces between the atoms and therefore perturb vibrational modes in different ways. ${ }^{24,25}$ The increase of the extinction coefficient in this frequency range has been calculated and differences concerning the light polarization have been predicted in a simple model. ${ }^{9}$ However, difficulties in the observation of a polarization dependence of the terahertz absorption was reported. Our data clearly represent distinct differences in the terahertz absorption for the two polarization orientations.

The hydration dynamics is traced by taking terahertz spectra and simultaneously changing the relative humidity to which the sample is exposed. We investigated the dehydration dynamics of salmon and calf thymus DNA samples with the helical axis aligned parallel or perpendicular to the terahertz beam polarization. Although the spectra of Fig. 1 show some spectral differences for the two polarizations, the temporal changes of the absorption under dehydration are qualitatively the same for the two polarizations. The dehydration process was monitored on samples which had been kept at $98 \%$ r.h. overnight, i.e., they are fully hydrated. By removing one of the mylar windows of the sample cell, the sample was exposed to dry air $(<10 \%$ r.h.) in the spectrometer box. Further sample and reference measurements were performed approximately every 2 min until no further change in the transmission of the sample was observed.

Figures 2(a) and 2(b) show the temporal evolution of the transmission change for the full frequency range from 0.1 up to $2.0 \mathrm{THz}$. The obtained spectra do not differ qualitatively from those shown in Fig. 1. The calf thymus DNA exhibits a broad absorption band around $400 \mathrm{GHz}$ which is most pronounced in the dehydrated state. However, sharp absorption 


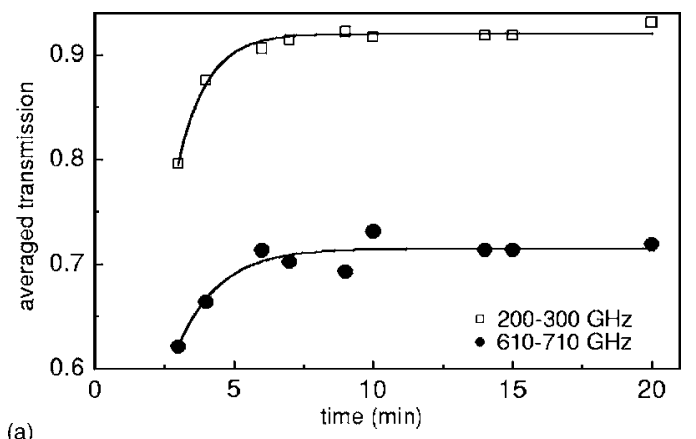

(a)

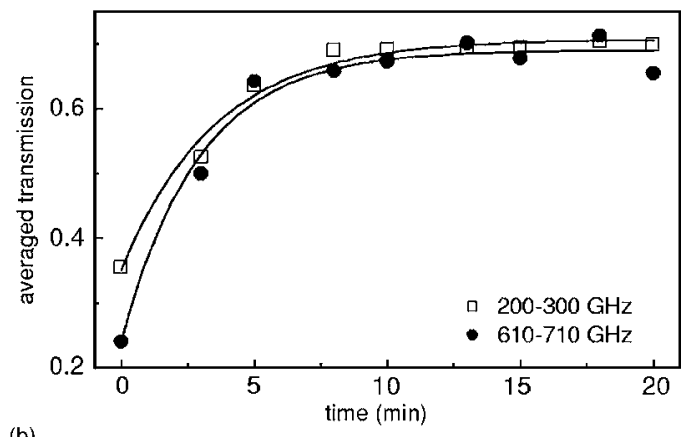

(b)

FIG. 3. Time dependence of the transmission averaged between 200-300 GHz and $610-710 \mathrm{GHz}$ for a salmon DNA film (a) and a calf thymus DNA film (b). The straight lines are fitted data.

bands are not expected for DNA with random sequence at room temperature. ${ }^{17}$ The sharp features reported in Ref. 10 could not be reproduced. A sharp feature which was observed at $1.7 \mathrm{THz}$ in Fig. 2(a) in the 3 min data after dehydration stems from residual water absorption in the setup. In the dehydration dynamics, a fast increase in transmission is observed as soon as the sample is exposed to dry air. We fit the function $T=T_{0}\left(1-e^{-\left(t-t_{0}\right) / \tau}\right)$ to the transmission traces averaged over $200-300 \mathrm{GHz}$ and over $610-710 \mathrm{GHz}$ for both films [Figs. 3(a) and 3(b)]. For the thinner salmon film, we find shorter time constants $(\tau=60 \mathrm{~s}$ at $200-300 \mathrm{GHz}$ and $84 \mathrm{~s}$ at $610-710 \mathrm{GHz}$ ) than for the thicker calf thymus film $(\tau=210 \mathrm{~s}$ at $200-300 \mathrm{GHz}$ and $174 \mathrm{~s}$ and at $610-710 \mathrm{GHz}$ ). After $10 \mathrm{~min}$ the absorption saturates.

The observed time scale for the conformational change is corroborated by Raman spectroscopy (see Fig. 4). The unpolarized Raman spectra exhibit the typical frequency bands between 600 and $900 \mathrm{~cm}^{-1}{ }^{26}$ The $807 \mathrm{~cm}^{-1}$ A-DNA marker appears in the salmon DNA film within 3 min after reducing the r.h.; $9 \mathrm{~min}$ after changing the r.h. the $A$-DNA marker is clearly visible. The observed time scale matches the rapid decrease in the absorption monitored by the terahertz time-domain spectra.

In conclusion, we use a high-speed ASOPS terahertz spectrometer to investigate the hydration dynamics of oriented DNA films. Differences in the absorption of perpendicular and parallel polarizations relative to the helix axis are observed. The transition from $B$ to $A$ conformation is monitored on a time scale of minutes. We believe that high-speed ASOPS based terahertz spectroscopy will be an important technique to monitor biological and chemical processes on short time scales.

The authors thank H. Schneider and M. Helm for the opportunity to perform Raman measurements and $\mathrm{U}$. Lucchesi for technical assistance. They thank F. Fontaine-

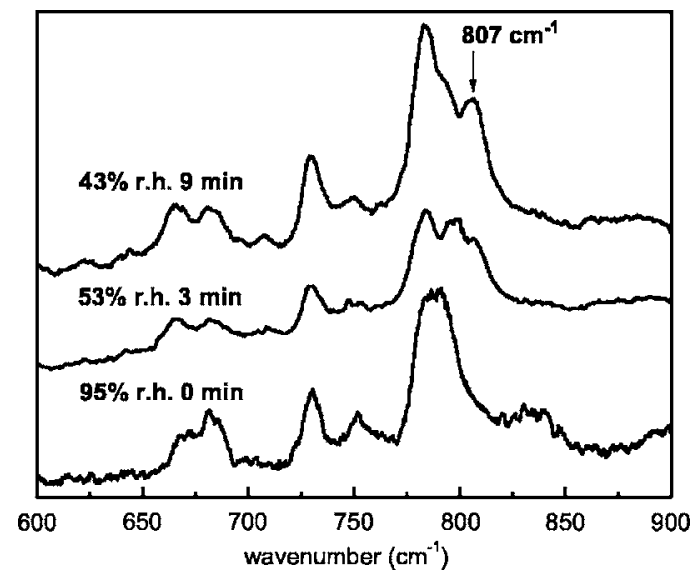

FIG. 4. Raman spectra of a salmon DNA film for different time after dehydration at different r.h., as given in the figure. The spectra are displaced vertically for clarity.

Vive, T. Forsyth, A. Rupprecht, and H. Grimm for help with producing the films and W. Welte and P. Seige for the help with the X-ray diffraction. This work is partially supported by the Ministry of Science, Research and the Arts of BadenWürttemberg and the Deutsche Forschungsgemeinschaft.

${ }^{1}$ T. E. Cheatham and P. A. Kollma, J. Mol. Biol. 259, 434 (1996).

${ }^{2}$ R. E. Dickerson, H. R. Drew, B. N. Conner, R. M. Wing, A. V. Fratini, and M. L. Kopka, Science 216, 475 (1982).

${ }^{3}$ J. M. Vargason, K. Henderson, and P. S. Ho, Proc. Natl. Acad. Sci. U.S.A. 98, 7265 (2001).

${ }^{4}$ W. Fuller, T. Forsyth, and A. Mahendrasingam, Philos. Trans. R. Soc. London, Ser. B 359, 1237 (2004).

${ }^{5}$ S. A. Lee, A. Rupprecht, and Y. Z. Chen, Phys. Rev. Lett. 80, 2241 (1998).

${ }^{6}$ T. Weidlich, S. M. Lindsay, Qi Rui, A. Rupprecht, W. L. Peticolas, and G. A. Thomas, J. Biomol. Struct. Dyn. 8, 139 (1990).

${ }^{7}$ G. Maret, R. Oldenbourg, G. Winterling, K. Dransfeld, and A. Rupprecht, Colloid Polym. Sci. 257, 1017 (1979).

${ }^{8}$ H. Grimm, P. M. Gehring, S. M. Shapiro, R. Kahn, and A. Rupprecht, Physica B 213-214, 780 (1995).

${ }^{9}$ A. Wittlin, L. Genzel, F. Kremer, S. Häseler, A. Poglitsch, and A. Rupprecht, Phys. Rev. A 34, 493 (1986).

${ }^{10}$ T. Globus, D. L. Woolard, T. Khromova, T. W. Crowe, M. Bykhovskaia, B. L. Gelmont, J. Hesler, and A. C. Samuels, J. Biol. Phys. 29, 89 (2003).

${ }^{11}$ R. Parthasarathy, T. Globus, T. Khromova, N. Swami, and D. Woolard, Appl. Phys. Lett. 87, 113901 (2005).

${ }^{12}$ U. Heugen, G. Schwaab, E. Bründermann, M. Heyden, X. Yu, D. M. Leitner, and M. Havenith, Proc. Natl. Acad. Sci. U.S.A. 103, 12301 (2006).

${ }^{13}$ J. Xu, K. W. Plaxco, and S. J. Allen, Protein Sci. 15, 1175 (2006).

${ }^{14}$ B. Ferguson and X.-C. Zhang, Nat. Mater. 1, 26 (2002).

${ }^{15}$ A. G. Markelz, A. Roitberg, and E. J. Heilweil, Chem. Phys. Lett. 320, 42 (2000).

${ }^{16}$ M. Brucherseifer, M. Nagel, P. Haring Bolivar, H. Kurz, A. Bosserhof, and A. Büttner, Appl. Phys. Lett. 77, 4049 (2000).

${ }^{17}$ B. M. Fischer, M. Walther, and P. U. Jepsen, Phys. Med. Biol. 47, 3807 (2002).

${ }^{18}$ A. Rupprecht, Biotechnol. Bioeng. 12, 93 (1970), and references therein.

${ }^{19}$ A. Rupprecht, Biochem. Biophys. Res. Commun. 12, 163 (1963).

${ }^{20}$ A. Bartels, R. Cerna, C. Kistner, A. Thoma, F. Hudert, C. Janke, and T. Dekorsy, Rev. Sci. Instrum. 78, 035107 (2007).

${ }^{21}$ A. Dreyhaupt, S. Winnerl, T. Dekorsy, and M. Helm, Appl. Phys. Lett. 86, 121114 (2005).

${ }^{22}$ Q. Wu and X.-C. Zhang, Appl. Phys. Lett. 71, 1285 (1997).

${ }^{23}$ Z. Kutnjak, C. Filipič, R. Podgornik, L. Nordenskiöld, and N. Korolev, Phys. Rev. Lett. 90, 098101 (2003).

${ }^{24}$ J. M. Eyster and E. W. Prohofsky, Phys. Rev. Lett. 38, 371 (1977).

${ }^{25}$ J. M. Eyster and E. W. Prohofsky, Biopolymers 16, 965 (1977).

${ }^{26}$ S. M. Lindsay, S. A. Lee, J. W. Powell, T. Weidlich, C. Demarco, G. D. Lewen, and N. J. Tao, Biopolymers 27, 1015 (1988). 